\title{
Same day comparison of PET/CT and PET/MR in patients with cardiac sarcoidosis
}

\author{
G. Wisenberg, MD, ${ }^{\text {a,f }}$ J. D. Thiessen, PhD, ${ }^{\mathrm{b}, \mathrm{e}}$ W. Pavlovsky, MD, ${ }^{\mathrm{c}}$ J. Butler, BSc, ${ }^{\mathrm{d}, \mathrm{e}}$ \\ B. Wilk, MSc, ${ }^{\mathrm{e}}$ and F. S. Prato, $\mathrm{PhD}^{\mathrm{b}, \mathrm{e}}$ \\ a Departments of Medicine, Medical Imaging, and Medical Biophysics, Western University, \\ London, ON, Canada \\ b Departments of Medical Biophysics, Medical Imaging and Physics and Astronomy, Western \\ University, London, ON, Canada \\ c Department of Medical Imaging, Western University, London, ON, Canada \\ d Division of Nuclear Medicine, St. Joseph's Hospital, London, ON, Canada \\ e Lawson Health Research Institute, London, ON, Canada \\ f MyHealth Centre, Arva, ON, Canada
}

Received Aug 26, 2018; Revised Dec 11, 2018

doi: $10.1007 / \mathrm{s} 12350-018-01578-8$

Background. Inflammatory cardiac disorders, in particular, sarcoidosis, play an important role in left ventricular dysfunction, conduction abnormalities, and arrhythmias. In this study, we compared the imaging characteristics and diagnostic information obtained when patients were imaged sequentially with PET/CT and then with hybrid PET/MRI on the same day following a single ${ }^{18}$ F-FDG injection.

Methods. Ten patients with known or suspected sarcoidosis underwent imaging in sequence of (a) ${ }^{99 m}$ Tc-MIBI, (b) ${ }^{18}$ F-FDG with PET/CT, and (c) ${ }^{18}$ F-FDG with 3T PET/MRI. Images were compared quantitatively by determination of $S U V_{\max }$ and $S U V$ on a voxel by voxel basis, and qualitatively by two experienced observers.

Results. When both platforms were compared quantitatively, similar data for the evaluation of enhanced ${ }^{18}$ F-FDG uptake were obtained. Qualitatively, there were (1) several instances of normal perfusion with delayed enhancement and/or focal ${ }^{18}$ F-FDG uptake, (2) comparable enhanced ${ }^{18}$ F-FDG uptake on PET/CT vs. PET/MRI, and (3) diversity in disease patterns with delayed enhancement only, increased ${ }^{18}$ F-FDG uptake only, or both.

Conclusion. In this limited patient study, PET/CT and PET/MR provided similar diagnostic data for ${ }^{18}$ F-FDG uptake, and the concurrent acquisition of MR images provided further insight into the disease process. (J Nucl Cardiol 2020;27:2118-29.)

Key Words: Cardiac sarcoidosis • positron emission tomography $\bullet$ magnetic resonance imaging • PET/CT • PET/MR • ${ }^{18}$ F-FDG

Electronic supplementary material The online version of this article (https://doi.org/10.1007/s12350-018-01578-8) contains supplementary material, which is available to authorized users.

The authors of this article have provided a PowerPoint file, available for download at SpringerLink, which summarizes the contents of the paper and is free for re-use at meetings and presentations. Search for the article DOI on SpringerLink.com.

Funding The Ontario Research Fund (ORF), the Canadian Foundation for Innovation (CFI), Bayer (Gadovist), and London X-ray Associates.
Reprint requests: G. Wisenberg, MD, MyHealth Centre, 21589 Richmond Street, Arva, ON NOM 1C0, Canada; gerald.wisenberg@ lawsonimaging.ca $1071-3581 / \$ 34.00$

Copyright (C) 2018 The Author(s) 


\begin{tabular}{|ll|}
\hline \multicolumn{2}{|l|}{ Abbreviations } \\
LV & Left ventricle \\
LVEF & Left ventricular ejection fraction \\
PET & Positron Emission Tomography \\
CT & Computed Tomography \\
MRI & Magnetic Resonance Imaging \\
${ }^{18}$ F-FDG & Fluorine-18 fluorodeoxyglucose \\
LGE & Late Gadolinium Enhancement \\
\hline
\end{tabular}

See related editorial, pp. 2130-2134

\section{INTRODUCTION}

Imaging has assumed an important role in diagnosis and guiding of therapeutic decisions in the setting of known or suspected sarcoidosis and cardiac inflammatory disorders. Cardiac involvement with sarcoidosis can lead to left ventricular dysfunction, conduction disturbances, and ventricular arrhythmias, ${ }^{1-5}$ related to active inflammation and/or scar. ${ }^{6}$ The presence of late Gadolinium enhancement in sarcoid patients confers a much higher risk of malignant ventricular arrhythmias. ${ }^{7}$ Cardiac involvement in sarcoidosis may affect up to $25 \%$ of patients based on pathology data. ${ }^{8,9}$

Co-incident with the understanding of the importance of sarcoidosis has been the development of hybrid imaging systems which combine Positron Emission Tomography (PET) with either Computed tomography (CT) or Magnetic Resonance Imaging (MRI). PET is ideally suited to assess active macrophage-mediated inflammation using ${ }^{18}$ F-FDG. ${ }^{10,11}$ Both M1 (pro-inflammatory macrophages) and M2 (anti-inflammatory macrophages) actively sequester ${ }^{18} \mathrm{~F}$-FDG, ${ }^{12,13}$ with evidence of a 20:1 ratio favoring sequestration by M1 macrophages. ${ }^{14}$ Inflammation can be detected by ${ }^{18} 18 \mathrm{~F}$ FDG imaging, provided myocardial ${ }^{18} \mathrm{~F}-\mathrm{FDG}$ uptake is selectively suppressed. When ${ }^{18}$ F-FDG-PET is combined with $\mathrm{CT}$, the $\mathrm{CT}$ provides attenuation correction, an anatomic reference, and unsuspected noncardiac findings. MRI not only provides the above, but also tissue characterization for the detection of scar and edema. When PET and MRI are combined, their geographic relationship (scar/edema and inflammation) can be ascertained. ${ }^{15}$ As such, hybrid PET/MRI may be more useful in managing individual patients, and on a broader scale, understanding the relationship and evolution of these processes may enhance our knowledge of the pathophysiology of sarcoidosis.
As a preliminary attempt to compare image characteristics and diagnostic details of PET/CT and PET/ MRI, we performed a pilot study of 10 patients with known or suspected cardiac sarcoidosis. ${ }^{10}$ All patients were imaged initially with PET/CT and then PET/MRI on the same day, following a single bolus injection of ${ }^{18}$ F-FDG.

\section{METHODS}

\section{Patient Selection}

Patients participated if they were to undergo an ${ }^{18}$ F-FDG PET/CT scan for assessment of active cardiac inflammation when there was a suspicion of sarcoidosis or in patients with established biopsy proven extracardiac sarcoid. Patients were consecutive patients who agreed to participate in the protocol and who did not have a cardiac defibrillator. Of 53 scans ordered during that time interval, 37 patients had defibrillators, and three declined participation, and three could not be imaged for technical reasons (size, body piercing). The remaining ten consented and form the cohort of the study.

\section{Imaging Protocols}

A summary of the imaging protocol is presented as Figure 1. Patients were asked (a) to fast for 12 hours prior to scanning, (b) to follow a high fat, low-carbohydrate, proteinpermitted diet the day before the scan, and (c) to present for the scanning session well hydrated.

For the perfusion study, SPECT imaging (Siemens Symbia T6 SPECT/CT) was performed using technetium $\left({ }^{99 \mathrm{~m}} \mathrm{Tc}\right)$ sestamibi. Approximately $1000 \mathrm{MBq}$ was injected intravenously. Scanning began after a delay of 30-60 minutes for blood clearance of the tracer. Acquisitions consisted of 60 static projections, 25 seconds/projection, reconstructed with an ordered subset expectation maximization (OSEM) algorithm, Butterworth filter ( 0.4 cycles/cm cutoff), and CT-based attenuation correction. Tomographic images were presented as polar maps using the Emory Cardiac Toolbox on a GE Xeleris workstation for review. Perfusion was only assessed in this manner. There was no PET perfusion study performed in these patients.

One hour prior to the scheduled scan, ${ }^{18} \mathrm{~F}$-FDG [5 MBq/ $\mathrm{kg}$ injected intravenously ( $<550 \mathrm{MBq}(15 \mathrm{mCi})$ maximum] was administered. Unfractionated Heparin ( 5 units $/ \mathrm{kg}$ ) was given intravenously 45 minutes before ${ }^{18} \mathrm{~F}-\mathrm{FDG}$, followed by an additional dose of Heparin (10 units $/ \mathrm{kg}$ ) 30 minutes after the first injection (or 15 minutes prior to ${ }^{18} \mathrm{~F}$-FDG administration).

${ }^{18} \mathrm{~F}-{ }^{18} \mathrm{~F}-\mathrm{FDG}$ whole-body (1-3 minutes/bed position) and thorax-only (10 minutes each) PET images (PET ${ }_{\text {WBCT }}$ and $\mathrm{PET}_{\mathrm{ThoCT}}$ ) were acquired on a PET/CT (GE Discovery VCT). 


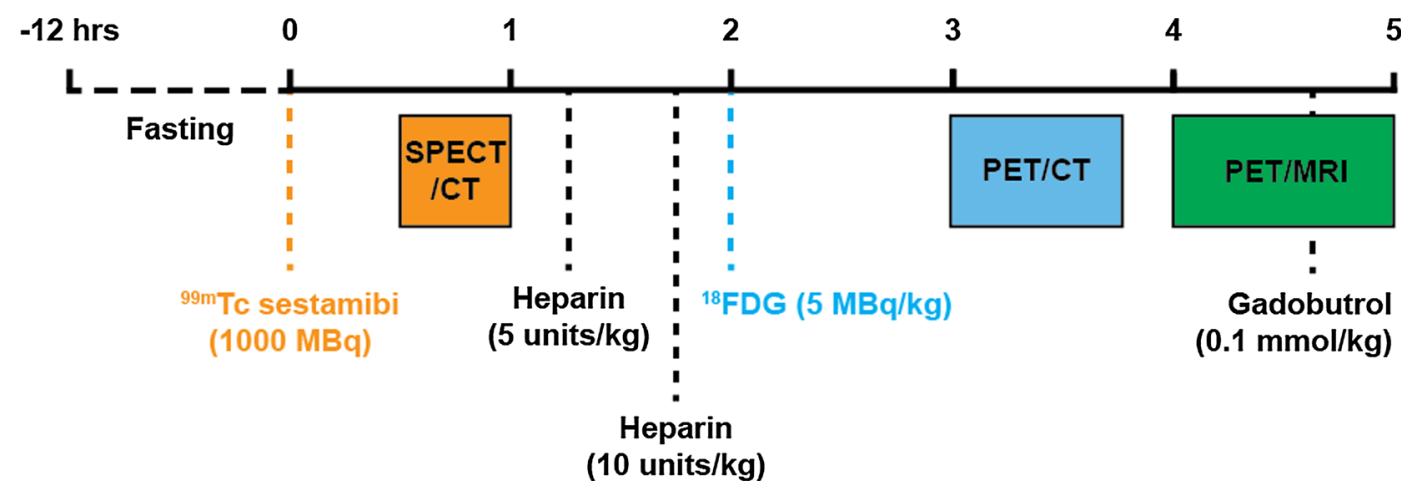

Figure 1. Schematic presentation of the imaging protocol.

PET/CT included a low-dose CT for attenuation correction (140 kVp, variable $\mathrm{mA})$. PET from the PET/CT was reconstructed using the vendor-provided OSEM algorithm with an axial resolution of $5.47 \mathrm{~mm}$ and slice thickness of $3.27 \mathrm{~mm}$.

Shortly after PET/CT, patients had thorax-only PET/MRI $\left(\mathrm{PET}_{\mathrm{MR}}\right)$ acquired on a Siemens Biograph $\mathrm{mMR}$ in a single bed position with a 3 Tesla magnet. A 25 minute PET scan was acquired during the first half of the PET/MR imaging session. PET images were reconstructed using the vendor-provided OSEM algorithm with 3 iterations, 21 subsets, an axial resolution of $2.09 \mathrm{~mm}$, slice thickness of $2.03 \mathrm{~mm}$, and $4 \mathrm{~mm}$ Gaussian filter.

MRI acquisitions during the PET/MRI scan included the vendor-provided 2-point Dixon MR-based attenuation correction (MRAC) at the start of each PET acquisition, thoracic T2weighted coronal and axial half-fourier acquisition single-shot turbo spin echo (HASTE), breath-hold cine images using fast imaging with steady state precession (TrueFISP), and turbo inversion recovery magnitude (TIRM) images. Following a bolus injection of $0.1 \mathrm{mmol} / \mathrm{kg}$ gadobutrol (Bayer Gadovis $\mathrm{t}^{\circledR}$ ), late gadolinium enhancement (LGE) phase-sensitive inversion recovery (PSIR) and 3D inversion-recovery gradient-echo (3DLGE) images were acquired to image fibrosis in the left ventricle.

\section{IMAGE ANALYSIS}

\section{Clinical}

All images were reviewed in a nonblinded fashion by a dual-certified nuclear medicine/radiologist (WP) and cardiologist $(\mathrm{GW})$.

Initially, all the individual perfusion and corresponding ${ }^{18} \mathrm{~F}$-FDG PET/CT scans were reviewed. The presence or absence of perfusion defects, and the extent, and location of such defects were determined by visual analysis. Then, the presence of enhanced ${ }^{18} \mathrm{~F}-\mathrm{FDG}$ uptake was also established (only if it was regional, and significantly greater than blood pool activity). Diffuse uptake was suggestive of inadequate myocardial suppression.

Then, the PET/MRI images were reviewed on a separate day. The presence and extent of delayed enhancement was determined initially on the MR images. Following this, enhanced ${ }^{18} \mathrm{~F}$-FDG uptake on the PET/MRI images was determined through visual analysis. The PET and MRI images were then fused, and the relationship of any enhanced ${ }^{18} \mathrm{~F}-\mathrm{FDG}$ signal to the delayed enhancement was determined. LV wall motion was evaluated qualitatively for selected patients by a single observer (GW).

\section{Quantitative}

Standardized uptake values (SUV) were calculated using the injected dose, body weight, and decaycorrected time of injection. After determining the maximum SUV ( $\mathrm{SUV}_{\max }$ ) in the thoracic region (avoiding uptake in the liver and spine), regions of interest with elevated uptake were defined using a threshold equal to $0.5 \times \mathrm{SUV}_{\max }$ (3D Slicer 4.6.2). This was meant to determine the metabolically active volume (MAV) while accounting for variations in uptake over time and between patients. In addition, MAVs were calculated using fixed thresholds at SUV $>2.5$ (MAV 2.5) and SUV > 4 (MAV 4).

Left-ventricular end diastolic volumes (EDV), end systolic volumes (ESV), stroke volume (SV), and ejection fractions (LVEF) were determined using threshold-based segmentation with manual adjustments (as required) in short-axis cine images spanning the left ventricle (3D Slicer 4.6.2).

All measurements were tested for normality using the Shapiro-Wilk test, where the null hypothesis is a normal distribution and $P \leq 0.05$ indicates data is not normally distributed. 
In order to demonstrate the quantitative accuracy of $\mathrm{PET}_{\mathrm{MR}}$ vs $\mathrm{PET}_{\mathrm{CT}}$, linear regression and Pearson correlation coefficients were calculated for $\mathrm{SUV}_{\max }$ values from both $\mathrm{PET}_{\mathrm{MR}}$ vs $\mathrm{PET}_{\mathrm{WBCT}}$ and $\mathrm{PET}_{\mathrm{MR}}$ vs PETThoCT. A two-tailed paired $t$ test was used to compare $\mathrm{SUV}_{\max }$ values from both the PET/CT and PET/MRI. To measure voxel-wise correlation between SUV values, $\mathrm{PET}_{\text {ThoCT }}$ was manually aligned to $\mathrm{PET}_{\mathrm{MR}}$ by a dualcertified PET/MR technologist with additional guidance provided by both anatomical CT and MR images (3D Slicer 4.6.2). PET $_{M R}$ was then resampled to the $\mathrm{PET}_{\mathrm{ThoCT}}$ resolution and both PET volumes were cropped to a volume surrounding the heart before scatter and Bland-Altman plots were generated using the aligned $\mathrm{PET}_{\mathrm{ThoCT}}$ and $\mathrm{PET}_{\mathrm{MR}}$ voxel values. Linear regression and Pearson correlation correlations were also calculated between the LVEF and the $\mathrm{SUV}_{\text {max }}$ and the three estimates of MAV.

\section{Review of Prior MRI and/or CT Imaging}

If available, the results of prior studies were reviewed (in many cases there was a 2 month or greater time interval). All of these scans were performed on 1.5 Tesla systems.

\section{RESULTS}

A summary of patient characteristics are provided in Table 1, and of the MRI acquisition parameters in Table 2.

\section{Clinical Comparison}

The results of the clinical comparison are provided in Table 3 and Figures 2, 3, and 4. From these comparisons, we obtain the following observations:

Table 1. Summary of patient characteristics

\begin{tabular}{lllcll}
\hline Patient & Sex & Age & Weight (kg) & Biopsy & \multicolumn{1}{c}{ Prior history } \\
\hline 1 & M & 38 & 90.7 & NA & Nonischemic cardiomyopathy \\
2 & F & 66 & 56.7 & NA & Nonischemic cardiomyopathy \\
3 & F & 65 & 69.9 & + & Pulmonary sarcoid \\
4 & M & 69 & 122.0 & NA & Nonischemic cardiomyopathy \\
5 & M & 48 & 77.1 & + & Mediastinal sarcoid with pericarditis \\
6 & F & 59 & 107.5 & NA & Pulmonary sarcoid \\
7 & M & 70 & 89.8 & + (remote) & Pulmonary sarcoid \\
8 & F & 68 & 78.0 & NA & Syncope, nonsustained VT \\
9 & M & 63 & 108.9 & NS & Pulmonary and hilar adenopathy \\
10 & M & 58 & 95.7 & + (renal) & Pulmonary fibrosis \\
\hline
\end{tabular}

$N A$, not acquired; $N S$, nonspecific; +, positive biopsy

Table 2. MRI acquisition parameters

\begin{tabular}{|c|c|c|c|c|c|}
\hline & FOV $\left(\mathrm{mm}^{3}\right)$ & $\begin{array}{c}\text { Spatial } \\
\text { resolution }\left(\mathrm{mm}^{3}\right)\end{array}$ & $\begin{array}{l}\text { Motion } \\
\text { correction }\end{array}$ & $\begin{array}{l}\text { Acquisition } \\
\text { time/view }\end{array}$ & Views \\
\hline MRAC & $500 \times 328 \times 400$ & $2.6 \times 2.6 \times 3.2$ & None & $18 \mathrm{~s}$ & $3 D$ \\
\hline HASTE & $440 \times 440 \times 225$ & $1.72 \times 1.72 \times 6$ & $\mathrm{BH}$ & $5 s$ & Coronal, Axial \\
\hline TrueFISP & $286 \times 340 \times 6$ & $1.33 \times 1.33 \times 6$ & $\mathrm{BH}, \mathrm{ECG}$ & $10 \mathrm{~s}$ & $2 \mathrm{C}, 4 \mathrm{C}, \mathrm{SA}$ \\
\hline TIRM & $265 \times 340 \times 8$ & $1.33 \times 1.33 \times 8$ & $\mathrm{BH}, \mathrm{ECG}$ & $10 \mathrm{~s}$ & $2 \mathrm{C}, 4 \mathrm{C}, \mathrm{SA}$ \\
\hline PSIR & $217 \times 290 \times 8$ & $1.13 \times 1.13 \times 8$ & $\mathrm{BH}, \mathrm{ECG}$ & $6 \mathrm{~s}$ & $2 \mathrm{C}, 4 \mathrm{C}, \mathrm{SA}$ \\
\hline 3D-LGE & $250 \times 320 \times 130$ & $0.625 \times 0.625 \times 0.9$ & NE, ECG & $6-8 \mathrm{~min}$ & $3 \mathrm{D}$ \\
\hline
\end{tabular}

MRAC, MR-based attenuation correction; HASTE, half-fourier acquisition single-shot turbo spin echo; TrueFISP, true fast imaging with steady-state precession; TIRM, turbo inversion recovery magnitude; $P S I R$, phase-sensitive inversion recovery; $L G E$, late gadolinium enhancement; $F O V$, field of view; $B H$, breath-hold acquisition; $N E$, navigator echo for respiratory gating; $E C G$, electrocardiogram for cardiac gating or triggering; 2 C, 2-chamber view; $4 C$, 4-chamber view; $S A$, short-axis stack spanning the left ventricle from apex to base 
Table 3. Summary of SPECT, PET/CT, and PET/MRI readings

\begin{tabular}{|c|c|c|c|c|}
\hline Patient & SPECT & PET $_{C T}$ & PET $_{M R}$ & MRI \\
\hline $\begin{array}{l}1 \\
\text { (Fig. 2) }\end{array}$ & NP & $\begin{array}{l}\text { Increase in basal septum and } \\
\text { lateral wall. Extracardiac } \\
\text { uptake in hilum and great } \\
\text { vessels }\end{array}$ & Same as $\mathrm{PET}_{\mathrm{CT}}$ & $\begin{array}{l}\text { Diffuse patchy scar in } \\
\text { anterior, lateral, and } \\
\text { inferior walls }\end{array}$ \\
\hline 2 & NP & $\begin{array}{l}\text { Slight increase in lateral wall. } \\
\text { Increased thyroid uptake }\end{array}$ & $\begin{array}{l}\text { More obvious and } \\
\text { extensive uptake in } \\
\text { lateral wall, ascending } \\
\text { aorta, esophagus, and } \\
\text { right shoulder }\end{array}$ & $\begin{array}{l}\text { Extensive subendocardial } \\
\text { scar in inferior septum and } \\
\text { mid-inferior wall }\end{array}$ \\
\hline $\begin{array}{l}3 \\
\text { (Fig. 4) }\end{array}$ & $\begin{array}{l}\text { Anteroapical } \\
\text { PD }\end{array}$ & $\begin{array}{l}\text { No cardiac uptake. Uptake } \\
\text { in hilar nodes and thyroid }\end{array}$ & $\begin{array}{l}\text { No cardiac uptake. } \\
\text { Increased }{ }^{18} \text { F-FDG in } \\
\text { nodes, esophagus, } \\
\text { aorta, and left } \\
\text { subclavian }\end{array}$ & $\begin{array}{l}\text { Extensive mid-inferior and } \\
\text { inferior septal scar. } \\
\text { Transmural in some regions }\end{array}$ \\
\hline 4 & $\begin{array}{l}\text { Anterior and } \\
\text { lateral PD }\end{array}$ & $\begin{array}{l}\text { Increased }{ }^{18} \mathrm{~F}-\mathrm{FDG} \text { matching } \\
\mathrm{PD} \text { and in inferior wall }\end{array}$ & $\begin{array}{l}\text { More extensive uptake in } \\
\text { myocardium }\end{array}$ & $\begin{array}{l}\text { Mid-wall linear scar in septum } \\
\text { with no }{ }^{18} \text { F-FDG uptake in } \\
\text { that region. Extensive } \\
\text { edema involving anterior } \\
\text { wall, septum, and inferior } \\
\text { wall }\end{array}$ \\
\hline 5 & $\begin{array}{l}\text { DP in anterior } \\
\text { wall and } \\
\text { septum }\end{array}$ & $\begin{array}{l}\text { Increased }{ }^{18} \mathrm{~F}-\mathrm{FDG} \text { in basal } \\
\text { lateral wall. Increased } \\
\text { uptake in mediastinal } \\
\text { nodes }\end{array}$ & $\begin{array}{l}\text { Additional }{ }^{18} \mathrm{~F} \text {-FDG } \\
\text { uptake in septum, } \\
\text { apical and lateral wall. } \\
\text { Similar nodal uptake }\end{array}$ & No Gd-enhancement \\
\hline 6 & NP & $\begin{array}{l}\text { Increased }{ }^{18} \mathrm{~F}-\mathrm{FDG} \text { in basal } \\
\text { anterior wall and inferior } \\
\text { wall. Multiple extracardiac } \\
\text { sites including hilar, } \\
\text { mediastinal nodes, and } \\
\text { spine }\end{array}$ & $\begin{array}{l}\text { Similar but more } \\
\text { extensive uptake in } \\
\text { septum and lateral } \\
\text { wall. Similar nodal } \\
\text { uptake }\end{array}$ & $\begin{array}{l}\text { Distal anterolateral wall scar, } \\
\text { distal septal scar with }{ }^{18} \mathrm{~F} \text { - } \\
\text { FDG surrounding both scar } \\
\text { regions }\end{array}$ \\
\hline $\begin{array}{l}7 \\
\text { (Fig. 3) }\end{array}$ & NP & $\begin{array}{l}\text { Enhanced }{ }^{18} \mathrm{~F} \text {-FDG uptake in } \\
\text { basal septum and lateral } \\
\text { wall }\end{array}$ & $\begin{array}{l}\text { More extensive uptake in } \\
\text { lateral wall. Increased } \\
\text { uptake in lung }\end{array}$ & $\begin{array}{l}\text { Patchy distal septal } \\
\text { enhancement }\end{array}$ \\
\hline 8 & NP & Normal ${ }^{18}$ F-FDG & Normal ${ }^{18}$ F-FDG & $\begin{array}{l}\text { Lateral wall subendocardial } \\
\text { scar }\end{array}$ \\
\hline 9 & NP & $\begin{array}{l}\text { No cardiac uptake. } \\
\text { Extracardiac uptake in } \\
\text { hilum, lung, and aorta }\end{array}$ & Same as PET/CT & Small inferolateral scar \\
\hline 10 & NP & $\begin{array}{l}\text { No cardiac uptake. } \\
\text { Extracardiac uptake in } \\
\text { lungs, hilar, and } \\
\text { mediastinal nodes }\end{array}$ & Same as PET/CT & $\begin{array}{l}\text { Localized lateral } \\
\text { subendocardial scar }\end{array}$ \\
\hline
\end{tabular}




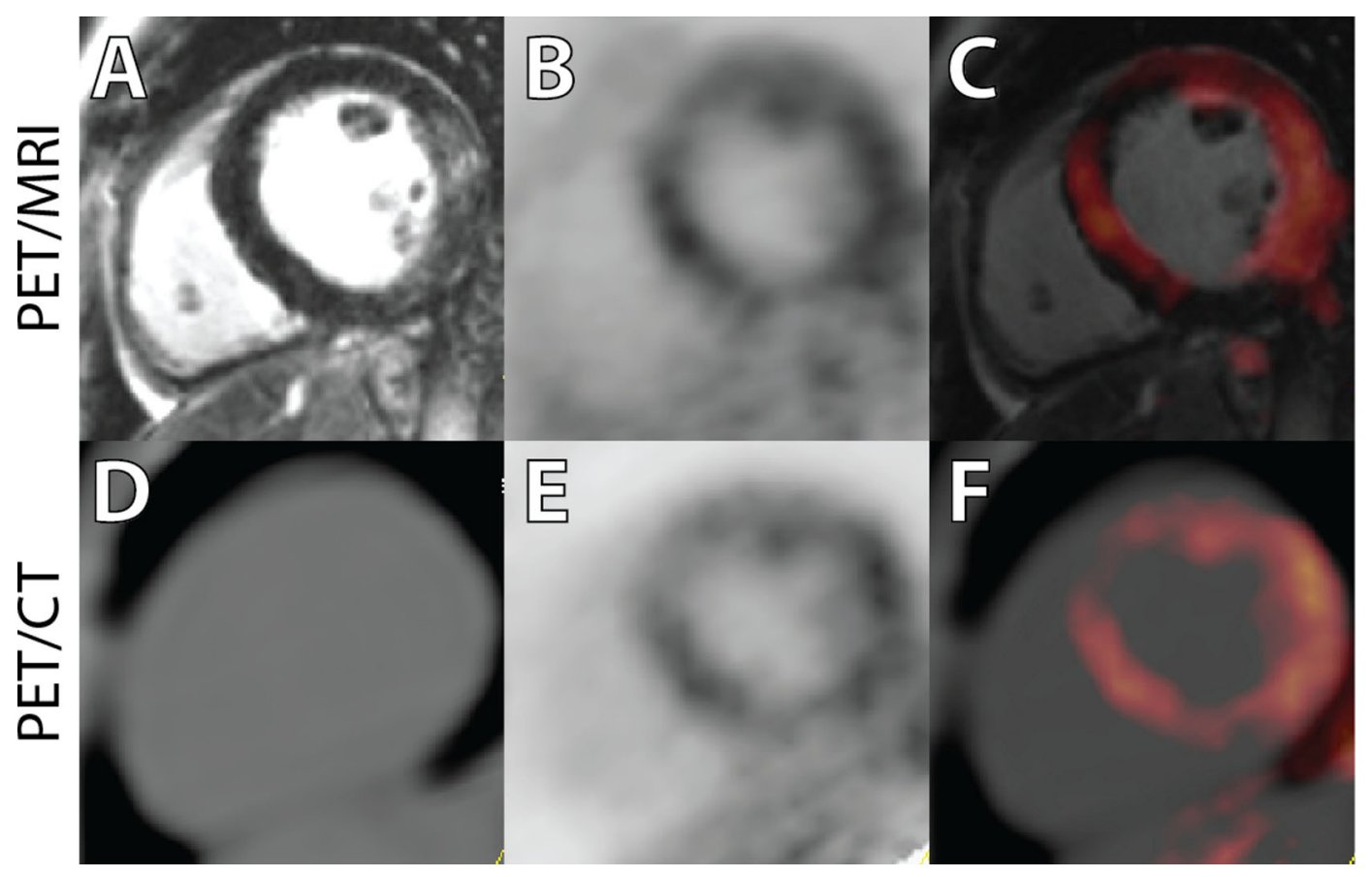

Figure 2. Images presented from Patient 1 with PET/MR (A-C) and PET/CT (D-F). In this patient, note the enhanced signal seen on the delayed enhancement MR images in the lateral wall (A), and the enhanced ${ }^{18} \mathrm{~F}$-FDG uptake in the septal, anterior, and lateral regions on both the PET/ $\mathrm{CT}$ and PET/MR images $(\mathbf{C}, \mathbf{F})$. Although this extensive uptake of ${ }^{18} \mathrm{~F}-\mathrm{FDG}$ could be interpreted as poor suppression, an increase in ${ }^{18} \mathrm{~F}-\mathrm{FDG}$ in the hilum and great vessels (not shown in these images) is supportive of this interpretation. This patient had an ejection fraction of $49 \%$ with mild global hypokinesis. There were no regional wall motion abnormalities.

(1) On comparison of ${ }^{18}$ F-FDG uptake on the PET/CT vs. PET/MRI, the presence/absence and the location of enhanced uptake were similar in all patients. The degree of contrast between enhanced uptake and background was greater on PET/MRI in all subjects with a positive ${ }^{18}$ F-FDG scan.

(2) Although perfusion abnormalities were seen in some patients, there were several instances of completely normal perfusion scans with enhanced regional and/ or patchy ${ }^{18}$ F-FDG uptake (pts $1,2,6$, and 7).

(3) Of the ten patients, eight had previously undergone a dedicated cardiac MRI on a 1.5 Tesla system. In all cases but one, there was a pattern of enhanced uptake suggestive of regional scar, with concordance between the MR findings on the dedicated MR and the PET/MR study.

(4) There were considerable divergence in the extent and patterns of sarcoid involvement, ranging from the presence of myocardial scar only, in pts 3,8 ,
9,10 (Figure 4), inflammation only (see Figure 3), or the two patterns present concurrently (Figure 2). In addition, several patients had significant extracardiac inflammation (hilar and mediastinal lymph nodes/great vessels) as indicated in Tables 1 and 3 .

\section{Quantitative Analysis}

$\mathrm{SUV}_{\max }$ values in the thoracic region determined by both PET/CT and PET/MRI were strongly correlated (Figure 5). Voxel-wise correlation of SUV values cropped to a region surrounding the heart in a single patient was also strong, but was influenced by the accuracy of the image registration and changing tissue uptake and blood concentration over time (Figure 6).

${ }^{18}$ F-FDG-PET results from all three acquisitions $\left(\mathrm{PET}_{\mathrm{WBCT}}, \mathrm{PET}_{\mathrm{ThoCT}}\right.$, and $\left.\mathrm{PET}_{\mathrm{MR}}\right)$ are summarized in Table 4. SUV ${ }_{\max }$ values were normally distributed with 


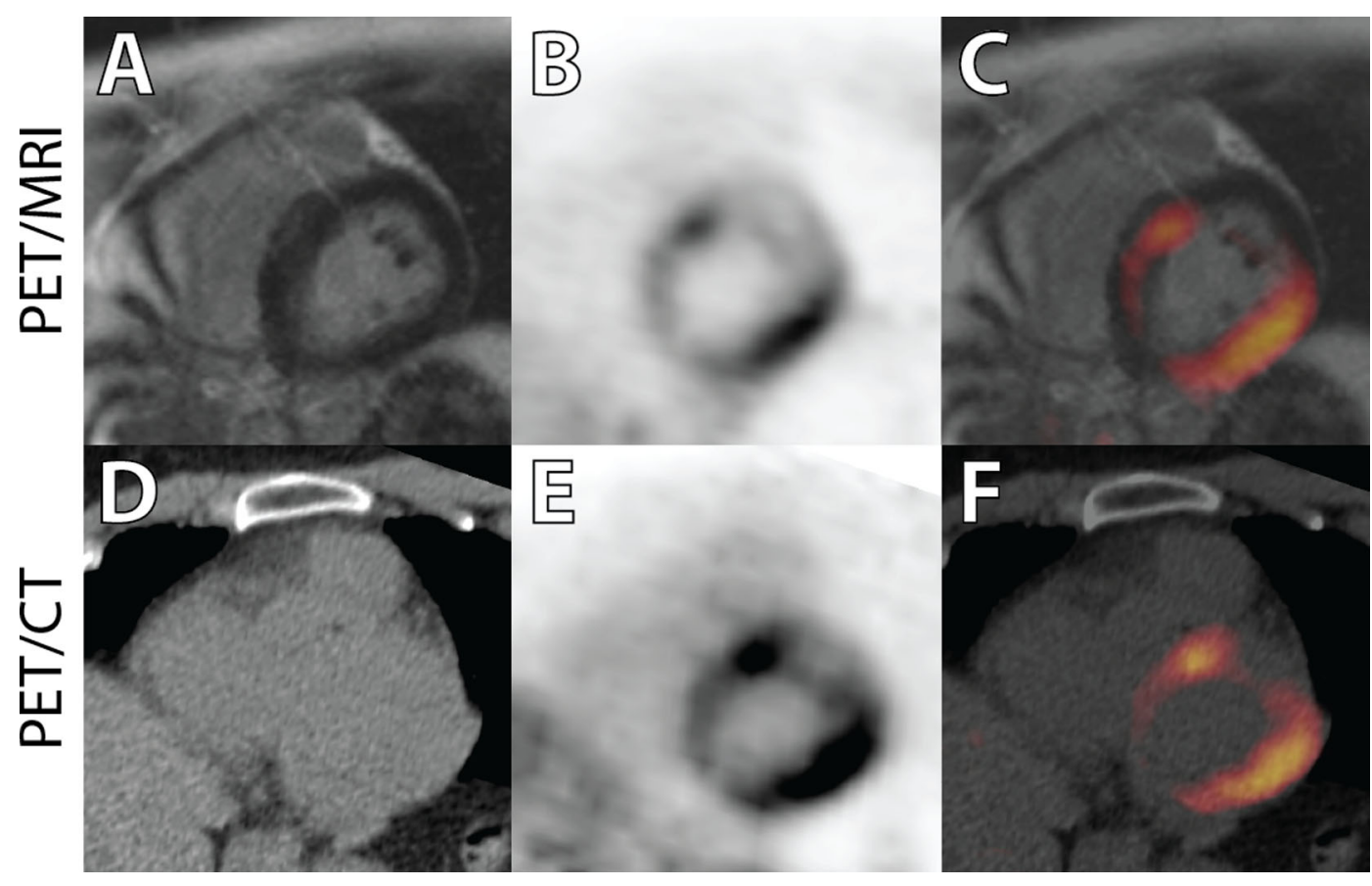

Figure 3. Images presented from patient 7 in a similar format as for Fig. 2. In this image, there is no area of signal enhancement on the delayed enhancement MR image (A). However, there is a marked increase in uptake, essentially transmural in the lateral wall and anterior septum $(\mathbf{B}, \mathbf{C})$. There is somewhat greater definition as to the extent of this uptake seen on the PET/MR images vs the PET/CT images $(\mathbf{E}, \mathbf{F})$. This patient's ejection fraction was $61 \%$ with moderate septal hypokinesis.

no significant differences between the PET data collected with PET/CT and PET/MR (Figures 5, 6).

Measurements of cardiac function are summarized in Table 5. Simultaneous acquisition of PET and MRI allowed for correlation of LVEF with PET measurements. Correlation between LVEF and $\mathrm{SUV}_{\max }$ was weak; however, there does appear to be increased correlation in the PET/MR between LVEF and MAV (Figure 7).

\section{DISCUSSION}

Imaging plays an important role in the diagnosis of cardiac inflammatory disorders, especially sarcoidosis. However, there are no universal guidelines as to which imaging tests should be performed routinely for initial screening in the absence of biopsy evidence. The most recent guidelines from $2014^{16}$ have suggested that it is probable that there is cardiac sarcoid if (a) there is biopsy evidence of the disease in an extracardiac site, and (b) one or more clinical criteria are met which include characteristic patterns for sarcoid on either MRI, ${ }^{18}$ F-FDG-PET, or gallium imaging, and other causes for these manifestations have reasonably been excluded. As such, the clinician is uncertain as to subjecting the patient to one or both imaging modalities, as MRI and PET focus on different aspects of the disease (MRI preferentially on scar/edema and ${ }^{18}$ F-FDG PET on macrophage-related inflammation). Combining both modalities simplifies decision making, but with the added expense of the hybrid unit. However, if both ${ }^{18} \mathrm{~F}$ FDG-PET and 3T LGE-MRI are ultimately needed, the total cost of performing these separately exceeds the cost of a single hybrid PET/MRI examination.

Our study has provided evidence that, strictly from an imaging perspective, as expected, nothing is lost when combining the two imaging modalities. In all cases, PET/MRI provided diagnostic quality ${ }^{18} \mathrm{~F}-\mathrm{FDG}$ 


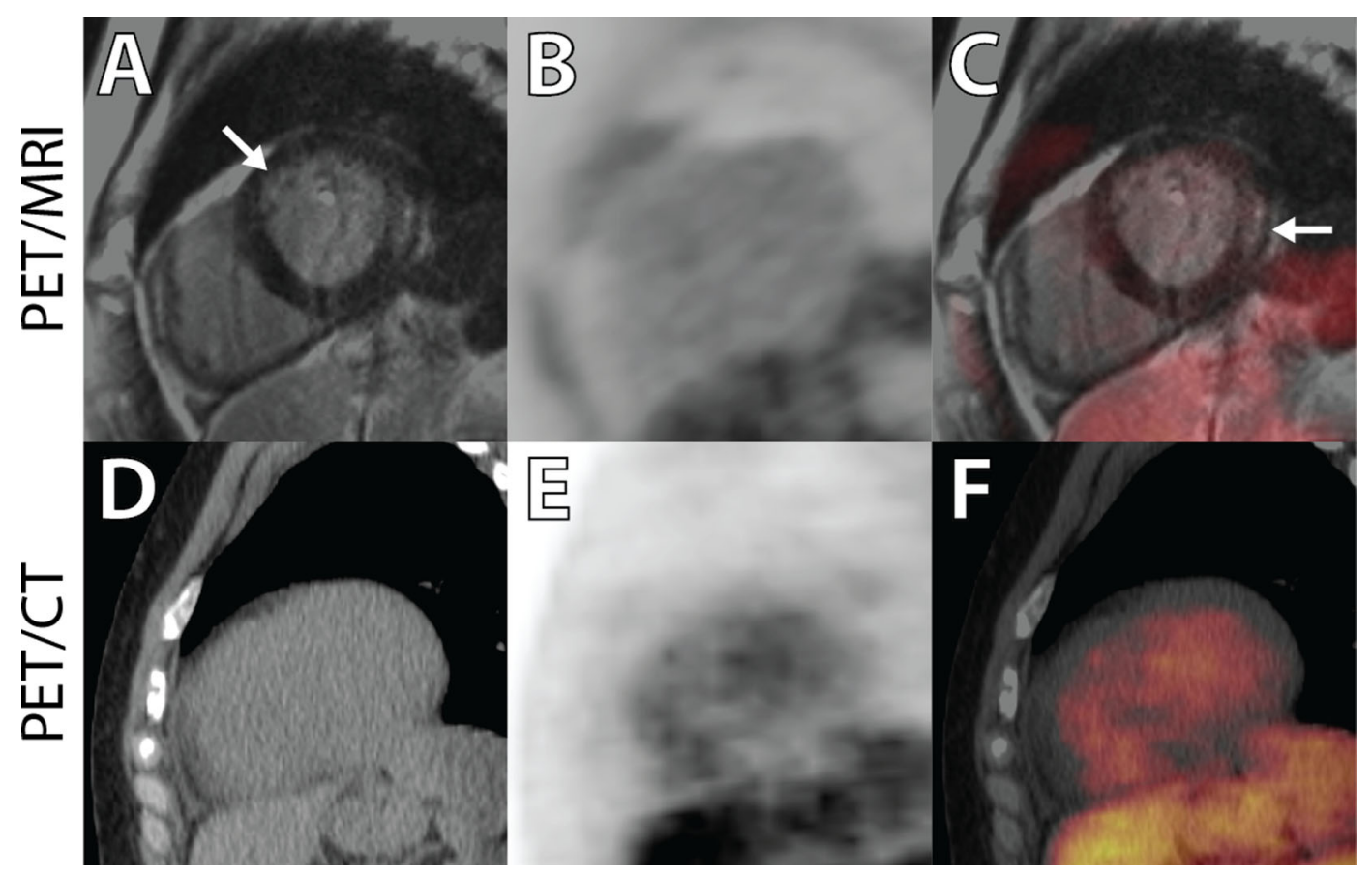

Figure 4. Images from patient three. In this case, an enhanced signal is seen on the delayed enhancement MR images in both mid-septum and lateral regions (A) [arrows]. There is no corresponding increase in ${ }^{18} \mathrm{~F}$-FDG signal on the PET images $(\mathbf{B}, \mathbf{C})$. Note the increased blood-pool activity on the PET/CT images that were taken approximately $2 \mathrm{~h}$ earlier (see Table 4), from the same single injection of ${ }^{18}$ F-FDG $(\mathbf{E}, \mathbf{F})$. The ejection fraction for this patient was $52 \%$ with mild inferoseptal hypokinesis.
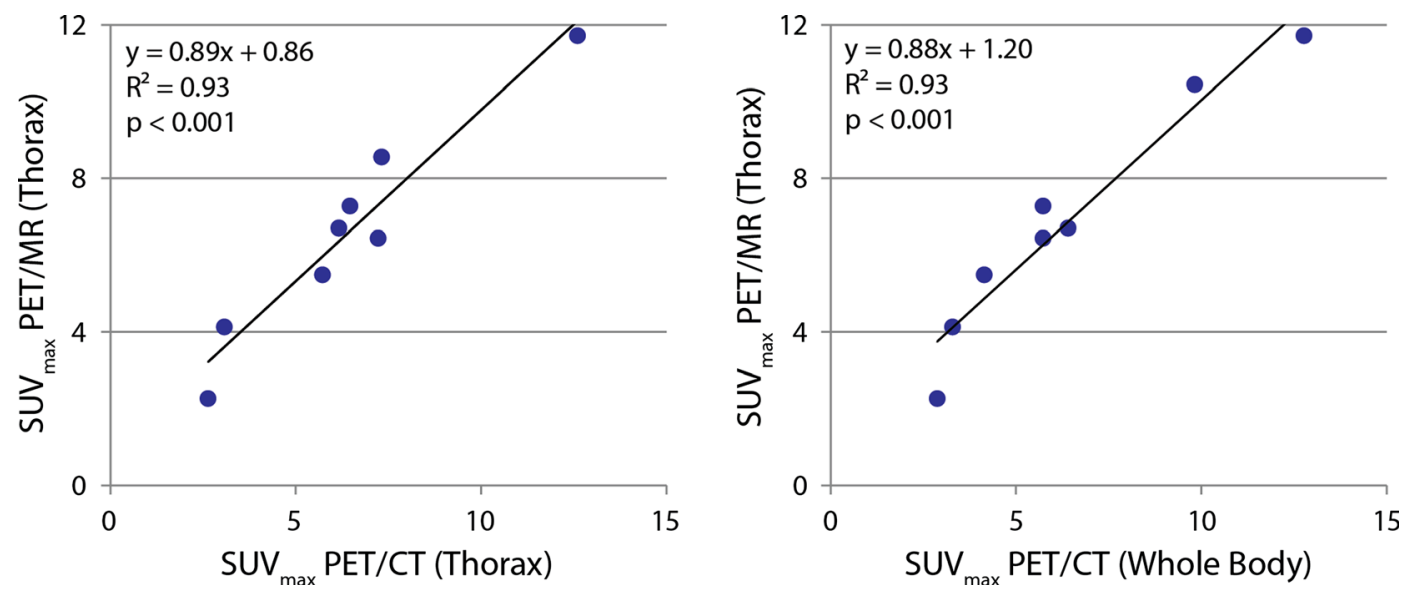

Figure 5. $\mathrm{SUV}_{\max }$ values determined with $\mathrm{PET} / \mathrm{MR}$ strongly correlating with both $\mathrm{SUV}_{\max }$ values determined with PET/CT acquired in a single bed position (left) and whole body (right). Note Patient eight was removed from comparison due to normal uptake in both PET/CT and PET/MR. One $\mathrm{SUV}_{\max }$ value was outside the axial FOV in the PET/CT (thorax) acquisition, and was removed from the comparison on the left. PET/CT (whole body) was not completed in another patient, and was removed from the comparison on the right. 

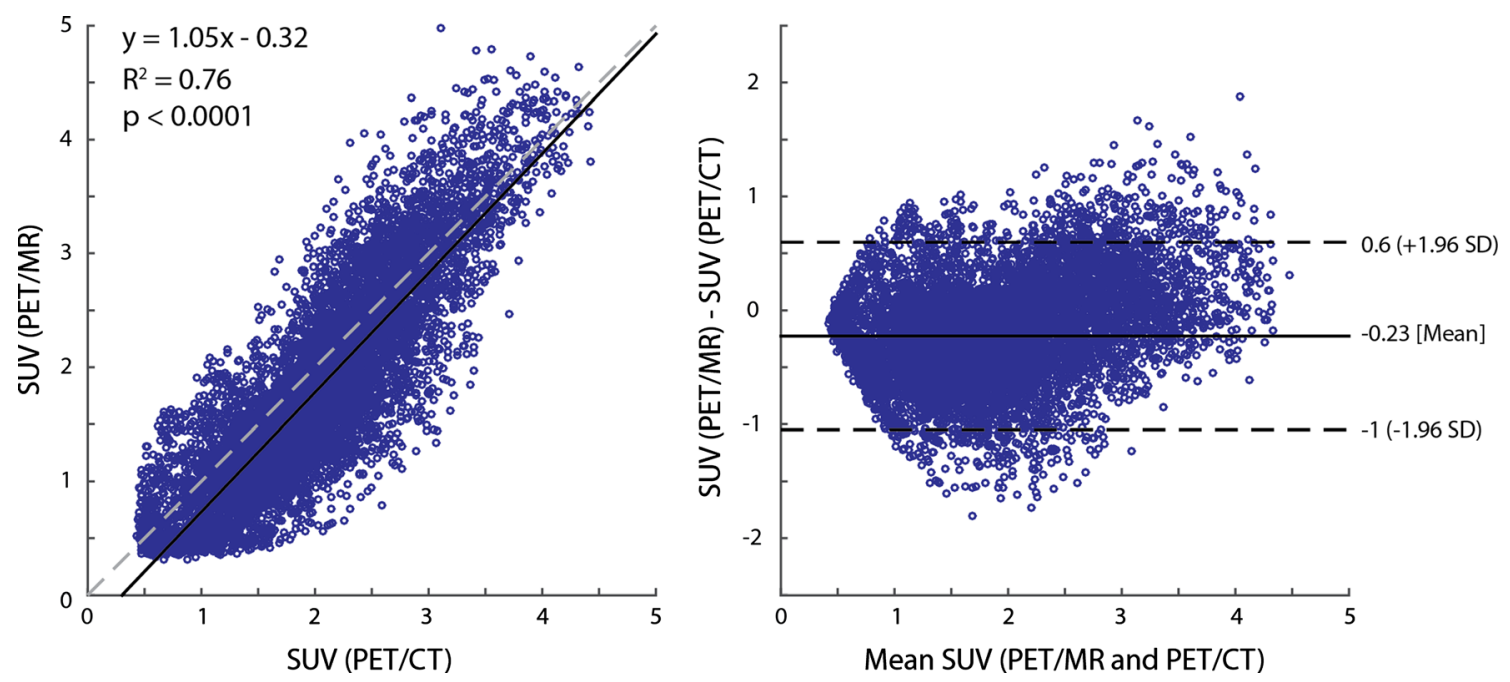

Figure 6. Voxel-wise comparison of co-registered and resampled SUV values from one PET/MR and PET/CT acquisition. Although correlation is strong, it is important to note that different times of acquisition and small registration errors will influence the correlation coefficient. Segmentation of the myocardium in the left ventricle was performed using the 3D-LGE MR data in this patient; however, this was not replicable due to patient motion in subsequent experiments.

images that were at least equal to or better than those obtained with either the PET/CT scan, and subjectively crisper MRI images than those obtained on a $1.5 \mathrm{~T}$ dedicated unit. If anything, the ${ }^{18}$ F-FDG images on the PET/MRI unit demonstrated, subjectively, improved image quality, with better definition of enhanced ${ }^{18} \mathrm{~F}$ FDG uptake. However, this may have been related to the fact that all the PET/MRI studies were performed after the PET/CT study, with greater clearance of tracer from blood, and that a longer acquisition time was used ( 25 vs 10 minutes) to compensate for decay.

Beyond its use for diagnostic purposes, imaging can provide prognostic information,which may be useful in guiding therapy, eg. immunosuppressive treatment, and/or an implantable defibrillator. An abnormal ${ }^{18}$ F-FDG-PET scan is associated with a greater risk of ventricular arrhythmias and death, ${ }^{5}$ as is delayed enhancement. ${ }^{7}$ Also, using serial examinations following treatment, a reduction in inflammation is associated with improved left ventricular function and prognosis, ${ }^{17}$ although we did not see any correlation between $\mathrm{SUV}_{\max }$ and LVEF in our study. Two recent studies have demonstrated divergent results with regards to the association of image findings with prognosis. A study reported by Vita showed that enhanced ${ }^{18}$ F-FDG uptake did not add prognostic value when combined with late gadolinium enhancement. ${ }^{18}$ However, a study by Wicks did suggest added value when the two modalities are combined. ${ }^{19}$

Although there was increased correlation between LVEF and metabolically active volumes in the thoracic region, given the small sample size and possible binomial distribution in some of the scatter plots, these results should be interpreted with caution. Nevertheless, this demonstrates the added value of acquiring PET and MRI data within the same system and at the same time.

\section{NEW KNOWLEDGE GAINED}

PET/MRI provides high-quality images in the evaluation of patients with suspected/known inflammatory disorders and potentially may be the diagnostic test of choice for evaluating these patients. 
Table 4. Summary of PET results

\begin{tabular}{|c|c|c|c|c|c|c|c|c|c|c|c|}
\hline \multirow[b]{2}{*}{$\mathbf{P t}$} & \multirow{2}{*}{\multicolumn{3}{|c|}{ Dose (MBq) }} & \multicolumn{4}{|c|}{ Start time after injection (m) } & \multicolumn{4}{|c|}{ SUV $_{\max }$} \\
\hline & & & & PET $_{\text {WB }}$ & \multicolumn{2}{|c|}{ PET Tho $_{\text {Tho }}$} & PET $_{\text {MR }}$ & \multicolumn{2}{|c|}{ PET WB $_{\text {W }}$} & PET $_{\text {Tho }}$ & \multirow{2}{*}{$\frac{\text { PET }_{\text {MR }}}{6.7}$} \\
\hline 1 & \multicolumn{3}{|c|}{300} & 136 & \multicolumn{2}{|l|}{146} & 210 & \multicolumn{2}{|c|}{6.4} & 6.2 & \\
\hline 2 & \multicolumn{3}{|c|}{260} & 62 & \multicolumn{2}{|l|}{94} & 133 & \multicolumn{2}{|c|}{2.9} & 2.6 & 2.3 \\
\hline 3 & \multicolumn{3}{|c|}{370} & 54 & \multicolumn{2}{|l|}{85} & 128 & \multicolumn{2}{|c|}{5.7} & 7.2 & 6.4 \\
\hline 4 & \multicolumn{3}{|c|}{628} & 56 & \multicolumn{2}{|l|}{94} & 139 & \multicolumn{2}{|c|}{3.3} & 3.1 & 4.1 \\
\hline 5 & \multicolumn{3}{|c|}{407} & NA & \multicolumn{2}{|l|}{94} & 131 & \multicolumn{2}{|c|}{ NA } & 7.3 & 8.5 \\
\hline 6 & \multicolumn{3}{|c|}{528} & 59 & \multicolumn{2}{|l|}{91} & 133 & \multicolumn{2}{|c|}{5.7} & 6.5 & 7.3 \\
\hline 7 & & 371 & & 81 & 115 & & 162 & 4.1 & & 5.7 & 5.5 \\
\hline 8 & & 415 & & 79 & 93 & & 141 & $\mathrm{~N}$ & & $\mathrm{~N}$ & $\mathrm{~N}$ \\
\hline 9 & & 542 & & 111 & 125 & & 165 & 12. & & 12.6 & 11.7 \\
\hline 10 & & 476 & & 76 & 83 & & 112 & 9.8 & & 6.5 & 10.4 \\
\hline Mean & & 430 & & 79 & 102 & & 145 & 6.3 & & 6.4 & 7.0 \\
\hline Std Dev & & 114 & & 28 & 20 & & 28 & 3.4 & & 2.9 & 2.9 \\
\hline & & & Suv $\stackrel{I}{>}$ & $\begin{array}{l}\text { MAV (cc) } \\
>0.5 \times \mathrm{SI}\end{array}$ & $\mathbf{U} \mathbf{V}_{\max }$ & & $\begin{array}{l}\text { MAV (cc) } \\
\text { iUV > } 2 .\end{array}$ & & & $\begin{array}{l}\text { MAV (cc) } \\
\text { SUV >4 }\end{array}$ & \\
\hline Pt & Dose & e (MBq) & PET $_{\text {WB }}$ & PET $_{\text {Tho }}$ & PET $_{\text {MR }}$ & PET $_{\text {WB }}$ & PET $_{\text {Tho }}$ & PET $_{\text {MR }}$ & PET $_{\text {WB }}$ & 3 PET Tho $_{\text {Tho }}$ & PET $_{M R}$ \\
\hline 1 & 300 & & 473 & 247 & 182 & 1000 & 504 & 361 & 129 & 86 & 90 \\
\hline 2 & 260 & & - & - & - & 1.3 & 0.4 & - & - & - & - \\
\hline 3 & 370 & & 59 & 12 & 37 & 93 & 34 & 78 & 13 & 7.6 & 16 \\
\hline 4 & 628 & & 1325 & 236 & 567 & 19 & 10 & 182 & - & - & 0.1 \\
\hline 5 & 407 & & NA & 26 & 23 & NA & 118 & 140 & NA & 19 & 28 \\
\hline 6 & 528 & & 96 & 28 & 33 & 212 & 88 & 136 & 15 & 7.5 & 20 \\
\hline 7 & 371 & & 200 & 27 & 37 & 75 & 51 & 53 & 0.8 & 3.3 & 5.4 \\
\hline 8 & 415 & & $\mathrm{~N}$ & $\mathrm{~N}$ & $\mathrm{~N}$ & $\mathrm{~N}$ & $\mathrm{~N}$ & $\mathrm{~N}$ & $\mathrm{~N}$ & $\mathrm{~N}$ & $\mathrm{~N}$ \\
\hline 9 & 542 & & 32 & 28 & 31 & 237 & 133 & 152 & 91 & 64 & 67 \\
\hline 10 & 476 & & 15 & 13 & 13 & 108 & 40 & 87 & 25 & 3.2 & 23 \\
\hline Mean & 430 & & $314^{*}$ & $77^{*}$ & $190 *$ & $218^{*}$ & $109 *$ & 149 & 46 & 27 * & 31 \\
\hline Std Dev & 114 & & 473 & 102 & 67 & 327 & 155 & 96 & 52 & 34 & 31 \\
\hline
\end{tabular}

$N A$, not acquired; $N$, normal uptake; $P E T_{W B}$, whole-body PET from PET/CT; $P E T_{T h o}$, thorax-only PET from PET/CT; $P E T_{M R}$, thoraxonly PET from PET/MR

${ }^{*} P \leq 0.05$ in Shapiro-Wilk normality test, where null-hypothesis $(P>0.05)$ is that the data is normally distributed

\section{LIMITATIONS}

T2/edema measurements were attempted by MRI but, as the patients were too exhausted, image quality was insufficient for analysis. Similarly, quantitative analysis of some MRI datasets was not possible due to motion artifacts. Further, the small sample size limits the ability to generalize the results, although the principal focus of the study was to compare the imaging characteristics of the two devices (PET/CT vs. PET/MR). The scans were interpreted in an unblinded fashion, but the quantitative comparison would not have been affected by this issue. Further, perfusion was assessed using Tcbased SPECT and not with a PET perfusion tracer. Finally, PET/MR was always delayed compared to PET/ $\mathrm{CT}$, changing the time from injection to imaging and decreasing the amount of cavitary blood activity and potentially affecting the SUV values.

Many potential patients were excluded from participation in this study because of the presence of implantable defibrillators. This will be an ongoing limitation of this hybrid technology unless the patient had an MRI-compatible unit implanted. 
Table 5. Summary of functional MRI results

\begin{tabular}{lllll}
\hline Patient & ESV (cc) & EDV (cc) & SV (cc) & LVEF (\%) \\
\hline 1 & 72.8 & 141.7 & 68.9 & 48.6 \\
2 & 28.0 & 55.9 & 27.9 & 49.9 \\
3 & 68.5 & 142.6 & 74.1 & 51.9 \\
4 & 223.1 & 280.3 & 57.1 & 20.4 \\
5 & NA & NA & NA & NA \\
6 & 31.4 & 89.5 & 58.1 & 64.9 \\
7 & 31.3 & 79.9 & 48.6 & 60.8 \\
8 & 61.5 & 119.7 & 58.2 & 48.6 \\
9 & 45.0 & 105.2 & 60.1 & 57.2 \\
10 & 58.6 & 116.2 & 57.5 & 49.5 \\
Mean & $68.9^{*}$ & $125.7^{*}$ & 56.7 & $50.2^{*}$ \\
Std Dev & 60.2 & 64.5 & 13.1 & 12.6
\end{tabular}

$E D V$, end diastolic volume; $E S V$, end systolic volume; SV, stroke volume; $L V E F$, left ventricular ejection fraction; $N A$, not acquired ${ }^{*} P \leq 0.05$ in Shapiro-Wilk normality test, where null-hypothesis $(P>0.05)$ is that the data is normally distributed
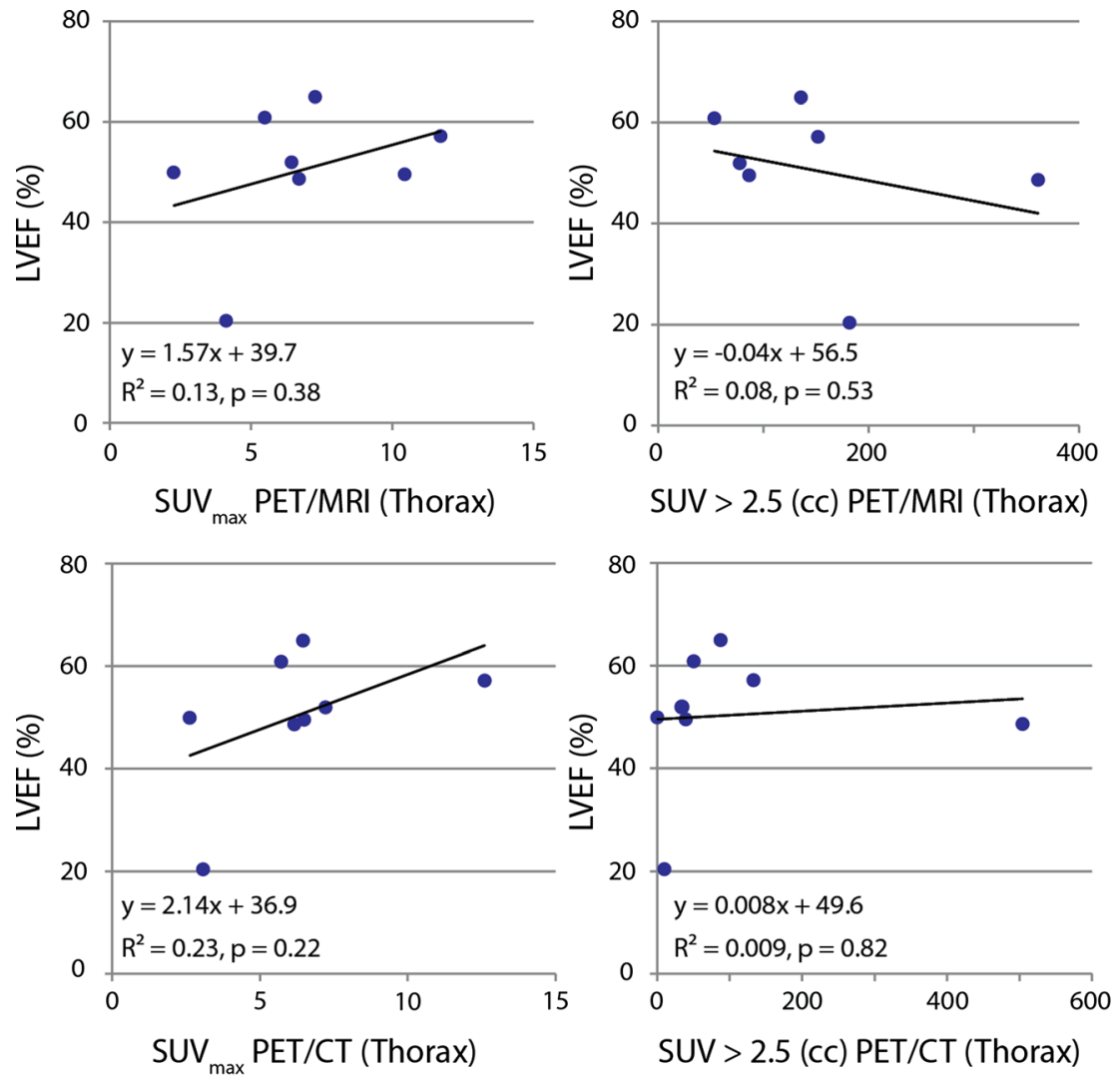
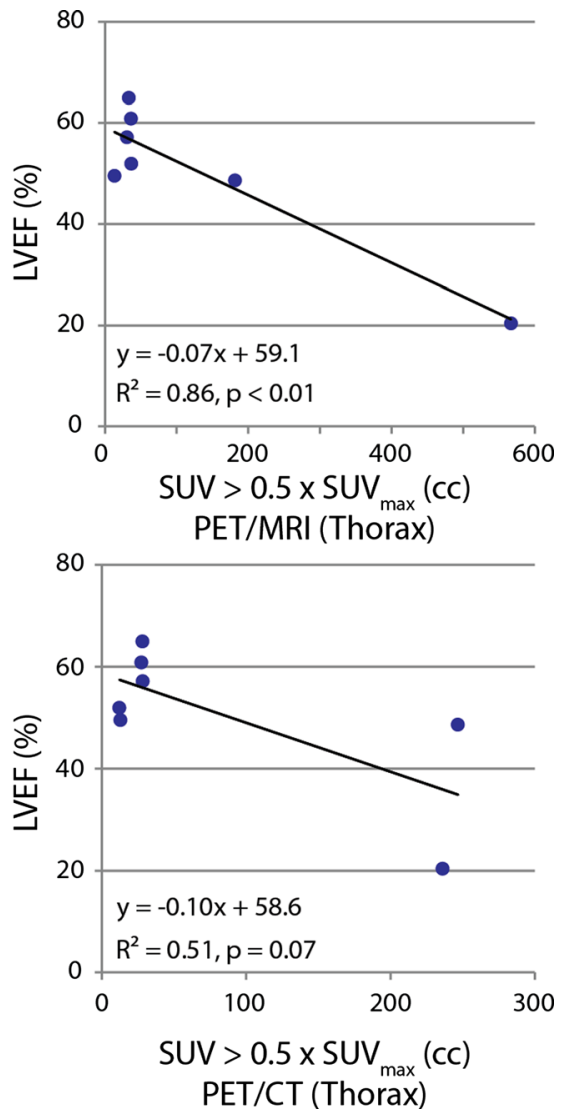

Figure 7. Left: weak correlation between LVEF and $\mathrm{SUV}_{\max }$. One advantage of PET/MR is the ability to measure functional MRI simultaneously with metabolic PET. Although these results should be interpreted with caution, we see an increased correlation, particularly in the PET/MR, between LVEF and metabolically active volumes in the thoracic region defined using a threshold of $\mathrm{SUV}>2.5$ (middle) and $\mathrm{SUV}>0.5 \times \mathrm{SUV}_{\max }$ (right). 


\section{CONCLUSIONS}

This study provides a small sample of the imaging capabilities and the potential role in patient management of advanced hybrid imaging with PET/MRI. Further studies are needed to validate the management and costbenefit aspects of this modality.

\section{Disclosure} article.

There are no disclosures from the authors related to this

\section{Open Access}

This article is distributed under the terms of the Creative Commons Attribution 4.0 International License (http://creativ ecommons.org/licenses/by/4.0/), which permits unrestricted use, distribution, and reproduction in any medium, provided you give appropriate credit to the original author(s) and the source, provide a link to the Creative Commons license, and indicate if changes were made.

\section{References}

1. Divakaran S, Singh A, Collins B, Vita T, Falk RH, Di Carli MF, et al. Role of imaging in evaluating infiltrative heart disease. Curr Treat Options Cardio Med. 2017;19:3-11.

2. Birnie DH, Nery PB, Ha AC, Beanlands RSB. Cardiac sarcoidosis. J Am Coll Cardiol. 2016;16:411-21.

3. Mehta D, Lubitz SA, Frankel Z, Wisnivesky JP, Einstein AJ, et al. Cardiac involvement in patients with sarcoidosis: diagnostic and prognostic value of outpatient testing. Chest. 2008;133: 1426-35.

4. Crouser ED, Ono C, Tran T, He X, Raman SV. Improved detection of cardiac sarcoidosis using magnetic resonance with myocardial T2 mapping. Am J Respir Crit Care Med. 2015;189:109-12.

5. Blankstein R, Osborne M, Naya M, Waller A, Kim CK, Murthy VL, et al. Cardiac positron emission tomography enhances prognostic assessments of patients with suspected cardiac sarcoidosis. J Am Coll Cardiol. 2014;63:329-36.

6. Greulich S, Deluigi CC, Gloekler S, Wahl A, Zürn C, Kramer U, et al. CMR imaging predicts death and other adverse events in suspected cardiac sarcoidosis. JACC Cardiovasc Imaging. 2013;6:501-11.

7. Hulten E, Agarwal V, Cahill M, Cole G, Vita T, Parrish S, et al. Presence of late gadolinium enhancement by cardiac magnetic resonance among patients with suspected cardiac sarcoidosis is associated with adverse cardiovascular prognosis: A systematic review and meta-analysis. Circ Cardiovasc Imaging. 2016;9:e05001.
8. Iwai K, Tachibana T, Takemura T, Matsui Y, Kitaichi M, Kawabata Y. Pathological studies on sarcoidosis autopsy. Epidemiological features of 320 cases in Japan. Acta Pathol Jpn. 1993;43:372-6.

9. Perry A, Vuitch F. Causes of death in patients with sarcoidosis. A morphologic study of 38 autopsies with clinicopathologic correlations. Arch Pathol Lab Med. 1995;119:167-72.

10. Osborne MT, Hulten EA, Murthy V, Skali H, Taqueti VR, Dorbala $\mathrm{S}$, et al. Patient preparation for cardiac fluorine-18 fluorodeoxyglucose positron emission tomography imaging of inflammation. J Nucl Cardiol. 2017;24:86-99.

11. Youseff G, Leung E, Mylonas I, Nery P, Williams K, Wisenberg G. The use of $18 \mathrm{~F}^{18}{ }^{18} \mathrm{~F}$-FDG PET in the diagnosis of cardiac sarcoidosis: a systematic review and meta-analysis including the Ontario experience. J Nucl Med. 2012;53:241-8.

12. Satomi T, Ogawa M, Mori I, Ishino S, Kubo K, Magata Y, et al. Comparison of contrast agents for atherosclerosis imaging using cultured macrophages. FDG versus ultrasmall superparamagnetic iron oxide. J Nucl Med. 2013;54:999-1004.

13. Jones HA, Cadwallader KA, White JF, Uddin M, Peters AM, Chilvers ER. Dissociation between respiratory burst activity and deoxyglucose uptake in human neutrophil granulocytes: implications for interpretation of ${ }^{18} \mathrm{~F}-18 \mathrm{~F}-\mathrm{FDG}$ PET images. J Nucl Med. 2002;43:652-7.

14. Thackeray J, Ludwig T, Ross L, Bankstahl J, Wester H, Bengel F. Targeting cardiovascular inflammation for imaging: Comparison of the uptake of multiple tracers in leukocyte subpopulations. J Nucl Med. 2017;58:302 (abstract).

15. White JA, Rajchl M, Butler J, Thompson RT, Prato FS, Wisenberg G. Active cardiac sarcoidosis: first clinical experience of simultaneous positron emission tomography-magnetic resonance imaging for the diagnosis of cardiac disease. Circulation. 2013;127:e639-41.

16. Birnie DH, Sauer WH, Bogun F, Cooper JM, Culver DA, Duvernoy CS, et al. Heart Rhythm Society expert consensus statement on the diagnosis and management of arrhythmias associated with cardiac sarcoidosis. Heart Rhythm. 2014;11(7):130523.

17. Osborne MT, Hulten EA, Singh A, Waller AH, Bittencourt MS, Stewart GC, et al. Reduction in 18F-fluorodeoxyglucose uptake on serial cardiac positron emission tomography is associated with improved left ventricular ejection fraction in patients with cardiac sarcoidosis. J Nucl Cardiol. 2014;21:166-74.

18. Vita T, Okada DR, Veillet-Chowdhury M, Bravo PE, Mullins E, Hulten E, et al. Complementary value of cardiac magnetic resonance imaging and positron emission tomography/computed tomography in the assessment of cardiac sarcoidosis. Circ Cardiovasc Imaging. 2018;11:e007030.

19. Wicks EC, Menezes LJ, Barnes A, Mohiddin SA, Sekhri N, Porter JC, et al. Diagnostic accuracy and prognostic value of simultaneous 18-F-fluorodeoxyglucose positron emission tomography/magnetic resonance imaging in cardiac sarcoidosis. Eur Heart J Cardiovasc Imaging. 2018;19(7):757-67.

Publisher's Note Springer Nature remains neutral with regard to jurisdictional claims in published maps and institutional affiliations. 